\title{
The Study Of Multifunction External Fixator Based On Stewart Platform
}

\author{
Guo Yue ${ }^{1}$, Zhang Lu², Wei Mengting ${ }^{2}$ and Sun $\mathrm{Hao}^{2, \mathrm{a}}$ \\ ${ }^{1}$ National Research Center for Rehabilitation Technical Aids, Beijing, China \\ ${ }^{2}$ School of Control Engineering, Hebei University of Technology, Tianjin, China
}

\begin{abstract}
The article develops the model of bone deformities, making 6-DOF Parallel Mechanism have widely applied to correction of deformities. The platform's positional direct solution is the posture of the motion platform. Malformation can be measured by X-ray, based on the space coordinate transformation can find the final posture of the motion platform. Regarding the reverse solution to platform kinematics the paper gives a quick arithmetic program, six actuators to realize motion requirements. For the computer-assisted fracture reduction, we produced an application interface.
\end{abstract}

Keywords: 6-DOF; parallel; positional direct solution; numerical method

\section{Introduction}

Stewart platform is a kind of 6-DOF parallel mechanism, which has a stable and compact structure and high precision[1-3].It is very suitable for those work situation where the work space is limited or the work space is not large enough but the load is very heavy[4].

Fig. 1 shows the femur correction process in use of Stewart platform. Firstly, doctors install the fixation frame through operation: first, put the bone blocks where the two ends of the site to be corrected fixed in the upper and lower platforms, then the platform's translation or rotation can drive the corresponding bone block translating and rotating, as shown in Fig.1A; second, install the 6 studdles and tighten them, making them in the state of unadjustable, as shown in Fig. 1B. Secondly, use X-ray film to measure the corresponding parameters, then put the parameters into Computer-Aid Software and get the lengths of the 6 studdles. Doctors can accomplish the orthopedic by rotating regulating nut to realize the elongation or decurtation of the 6 studdles, as shown in Fig $1 \mathrm{C}$.
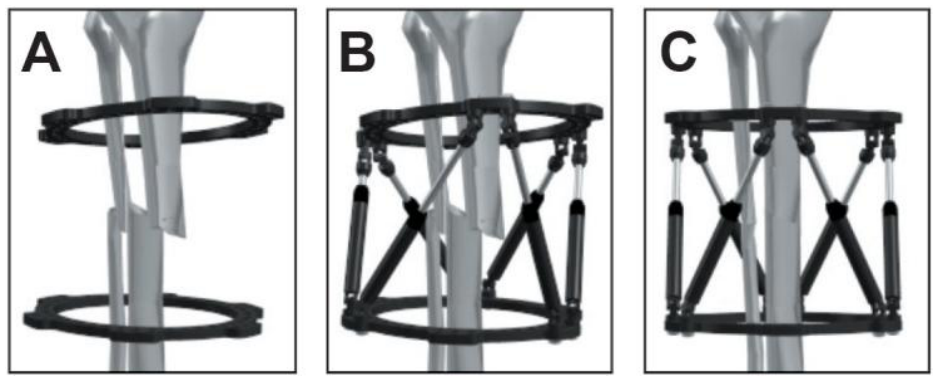

Fig 1.Femur correction diagram

\footnotetext{
a Corresponding author: sunhao@hebut.edu.cn
} 
Because of the particularity in structure of the St ewart platform,it is needed to regulate 6 studdles si multaneously in order to accomplish the movement of single degree of freedom[5]. However, if doctors make adjustment only depend on the subjective ex perience, it will be almost impossible to reach the goals, and even leading to more severe malformatio n.This paper studies on the Stewart platform Compli ance and Multifunction External Fixator correspondi ng software.

\section{Modelling}

We definite two coordinate systems, which are socle coordinate $\{\mathrm{B}\}$ and upper platform $\{\mathrm{P}\}$. The origin of the socle coordinate $\{\mathrm{B}\}$ is located in the center of the socle $\mathrm{O}_{\mathrm{b}}$, and the center of the upper platform $\mathrm{O}_{\mathrm{p}}$ is the origin of the upper platform coordinate $\{\mathrm{P}\}$, as shown in Fig.2.

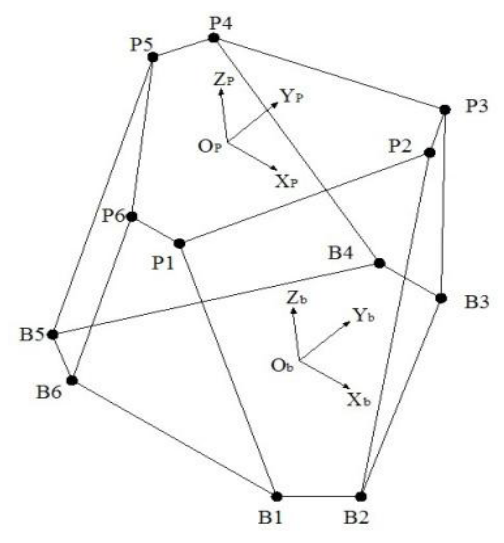

Fig 2. The definition of space

Set the moving platform $\{\mathrm{P}\}$ rotates the angle of $\gamma$ along $X_{b}$ axis, $\beta$ angle along $Y_{b}$ axis and $\alpha$ angle along $Z_{b}$ axis.Then we obtain the transfer matrix on coordinate $\{B\}$ along three axes in use of aforesaid definition.

$$
\begin{aligned}
R & =\left[\begin{array}{ccc}
c \alpha \cdot c \beta & c \alpha \cdot s \beta \cdot s \gamma-s \alpha \cdot c \gamma & c \alpha \cdot s \beta \cdot c \gamma+s \alpha \cdot s \gamma \\
s \alpha \cdot c \beta & s \alpha \cdot s \beta \cdot s \gamma+c \alpha \cdot c \gamma & s \alpha \cdot s \beta \cdot c \gamma-c \alpha \cdot s \gamma \\
-s \beta & c \beta \cdot s \gamma & c \beta \cdot c \gamma
\end{array}\right] \\
& =\left[\begin{array}{lll}
d_{11} & d_{12} & d_{13} \\
d_{21} & d_{22} & d_{23} \\
d_{31} & d_{32} & d_{33}
\end{array}\right] .
\end{aligned}
$$

Where $c \alpha=\cos \alpha, s \alpha=\sin \alpha, c \beta=\cos \beta, s \beta=\sin \beta$, $c \gamma=\cos \gamma, s \gamma=\sin \gamma$, We obtain the length of the studdle from Fig.2.

$$
l_{i}=\left|\overrightarrow{B_{i} P}\right|=\left|\overrightarrow{O_{b} O_{p}}+R \overrightarrow{B_{i} O_{p}}-\overrightarrow{O_{b} B_{i}}\right| i=1,2, \ldots, 6
$$

Therefore, if we obtain the position vector $\left[X_{P} Y_{P}\right.$ $\left.Z_{P}\right]^{T}$ and the vector of attitude angle $\left[\begin{array}{ccc}\alpha & \beta & \gamma\end{array}\right]^{\mathrm{T}}$ of the moving platform after orthopaedic,we can get the final lengths of six studdles by Eq.1. Firstly,put the six independent equations in Eq.2 into three groups i.e. $\left(l_{1}^{2}, l_{6}^{2}\right),\left(l_{2}^{2}, l_{5}^{2}\right)$ and $\left(l_{3}^{2}, l_{4}^{2}\right)$, then we carries out the calculation of addition or subtracting between the two equations in one group and obtain six independent equations in other forms.Expand each term in the equation and make use of relations as follows:

$$
\left.\begin{array}{c}
d_{11}^{2}+d_{21}^{2}+d_{31}^{1}=1 \\
d_{12}^{2}+d_{22}^{2}+d_{32}^{2}=1 \\
d_{11} d_{21}+d_{21} d_{22}+d_{31} d_{32}=0
\end{array}\right\}
$$

Then we get six independent equations as follows

$$
\begin{aligned}
\frac{l_{1}^{2}+l_{6}^{2}}{2}= & R_{P}^{2}+R_{B}^{2}+W-2 Y_{P} B_{1 Y}-2\left(d_{11} P_{1 X} B_{1 X}+d_{22} P_{1 Y} B_{1 Y}\right)+2 P_{1 Y}\left(D_{2} \bullet P\right) \\
& \frac{l_{1}^{2}-l_{6}^{2}}{4}=-X_{P} B_{1 X}-d_{21} P_{1 X} B_{1 Y}-d_{12} P_{1 Y} B_{1 X}+P_{1 X}\left(D_{1} \bullet P\right) \\
\frac{l_{2}^{2}+l_{5}^{2}}{2}= & R_{P}^{2}+R_{B}^{2}+W-2 Y_{P} B_{2 Y}-2\left(d_{11} P_{2 X} B_{2 X}+d_{22} P_{2 Y} B_{2 Y}\right)+2 P_{2 Y}\left(D_{2} \bullet P\right) \\
& \frac{l_{2}^{2}-l_{5}^{2}}{4}=-X_{P} B_{2 X}-d_{21} P_{2 X} B_{2 Y}-d_{12} P_{2 Y} B_{2 X}+P_{2 X}\left(D_{1} \bullet P\right) \\
\frac{l_{3}^{2}+l_{4}^{2}}{2}= & R_{P}^{2}+R_{B}^{2}+W-2 Y_{P} B_{3 Y}-2\left(d_{11} P_{3 X} B_{3 X}+d_{22} P_{3 Y} B_{3 Y}\right)+2 P_{3 Y}\left(D_{2} \bullet P\right) \\
& \frac{l_{3}^{2}-l_{4}^{2}}{4}=-X_{P} B_{3 X}-d_{21} P_{3 X} B_{3 Y}-d_{12} P_{3 Y} B_{3 X}+P_{3 X}\left(D_{1} \bullet P\right) . \\
D_{1}=\left\{d_{11},\right. & \left.d_{21}, d_{31}\right\}^{T}, D_{2}=\left\{d_{12}, d_{22}, d_{32}\right\}^{T}, W=X_{P}^{2}+Y_{P}^{2}+Z_{P}^{2} .
\end{aligned}
$$


After element elimination, there are three unknown quantities $X_{P}, Y_{P}, Z_{P}$ in the six equations, besides,each direction consine has been denoted by the function of $X_{P}$, $Y_{P}, Z_{P}$.Thus,if the lengths of six studdles (i.e.inputs) $l_{i}(i=1,2, \ldots, 6)$ are given,we can adopt optimization theory to solve this equation system, and obtain the initial position and pose of the upper platform.

Therefore, according to the malformation parameters and the installation framework parameters which are measured by doctors, we obtain the final position vector in use of coordinate frame and get the final lengths of six studdles through Eq.2.

\section{Bone model experiment}

Fig 3. Shows the demonstration framework of malfo rmation model of left femur.We can read the length $\mathrm{s}$ of six studdles as follows: $180 \mathrm{~mm}, 184 \mathrm{~mm}, 169 \mathrm{~mm}, 1$ $68 \mathrm{~mm}, 190 \mathrm{~mm}, 197 \mathrm{~mm}$. Through shooting orthophoria, we obtain the malformation parameters as follows:va lgus $10^{\circ}$,medial displacement $12.9 \mathrm{~mm}$. Besides,we get other malformation parameters from the side positio $\mathrm{n}$ image as follows:flexion $10^{\circ}$,anterior displacement $15 \mathrm{~mm}$,overlength $11 \mathrm{~mm}$. The installation parameters a re as follows:the center of the ring is located in 5 $\mathrm{mm}$ in front of the initial point,and breakpoint is 6 $5 \mathrm{~mm}$ to the proximal femur.Then put the aforeside parameters into the software,and we can obtain the treatment prescription.According to the final frame,w e adjust the lengths of six studdles as $166 \mathrm{~mm}, 178 \mathrm{~m}$ $\mathrm{m}, 173 \mathrm{~mm}, 167 \mathrm{~mm}, 158 \mathrm{~mm}, 162 \mathrm{~mm}$. Fig 4 . shows the e fficacy of orthopaedic.

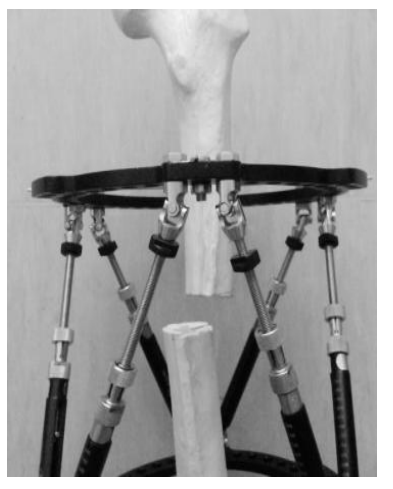

Fig 3a. Orthophoria image

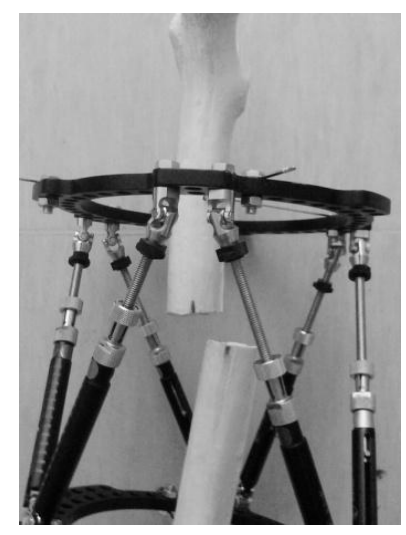

Fig 3b. Lateral image



Fig 4. The framework after orthopedic

\section{Clinical operation}

As shown in Fig 5.,the bone of Miss Jing's left tib ia is disjunct and has a angular deformity.Fig.6. sho ws the X-ray of orthophoria and lateral,after the me asurement,we can obtain the distortion parameters:va rus $50^{\circ}$, flexion $30^{\circ}$, internal rotation $25^{\circ}$; installation $\mathrm{p}$ arameters: proximal ring and distal ring are full-circ ular with a diameter of $155 \mathrm{~mm}$, the framework has a deviation of $10.8 \mathrm{~mm}$ to medial starting point,a devia tion of $46.2 \mathrm{~mm}$ to posterior starting point, the axial deviation is $80.0 \mathrm{~mm}$, and the external rotation of the framework is $15^{\circ}$; the initial lengths of six studdlesar e $178 \mathrm{~mm}, 190 \mathrm{~mm}, 166 \mathrm{~mm}, 147 \mathrm{~mm}, 127 \mathrm{~mm}, 145 \mathrm{~mm}$. Put these parameters into the software and obtain the fi nal lengths: $148 \mathrm{~mm}, 111 \mathrm{~mm}, 200 \mathrm{~mm}, 209 \mathrm{~mm}, 219 \mathrm{~mm}, 14$ $8 \mathrm{~mm}$.After some model experiments, the error rate of continuous orthopedic reaches $3 \mathrm{~mm}$ and $<1^{\circ}$. 




Fig 5.Before the operation

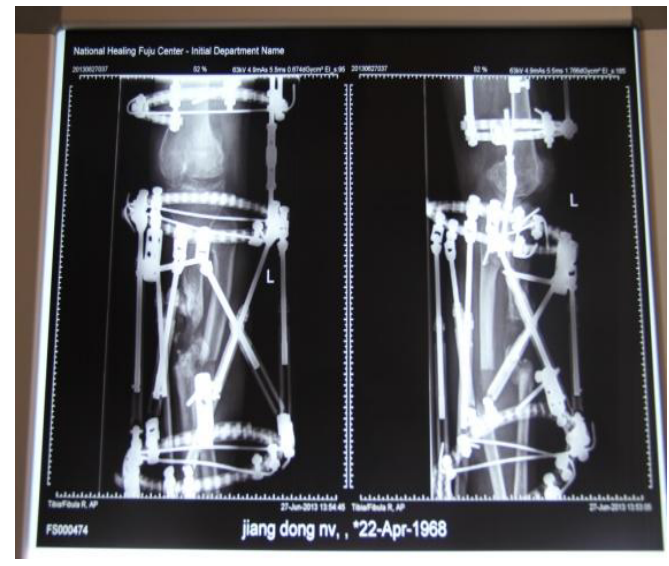

Fig 6.X-ray of the patient after installing Fixed frame

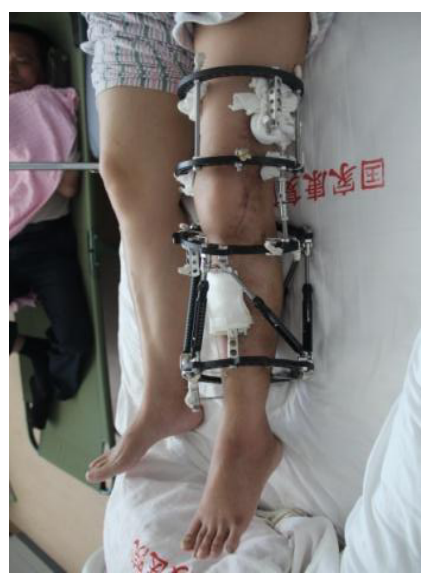

Fig 7.After the operation

\section{Conclusion}

Through the experiment of bone model, we can see the efficacy of disposable reduction is advisable. But because of some external factors such as patient's movement and so on, in order to reach the best efficacy, doctors should shoot X-ray after a period of time, remeasure distortion parameters, make a rejigger for treatment prescription, and use a method of continuous correction to minimize the deviation.

\section{Acknowledgements}

The research work was supported by Key Projects in the National Science \& Technology Pillar Program during the 12th Five-year Plan Period of China under Grant No. 2012BAI33B06.

\section{References}

[1] Zhen Huang: Spatial Kinematics. (China Machine Press, In Chinese, 1991)

[2] Guanglan Xia, Xiaoping Hu, and Caihua Li. China Science and Technology Information, vol.22, pp.28. (In Chinese, 2005)

[3] Zhen Huang, Yongsheng Zhao, and Tieshi Zhao: Higher Spatial Kinematics. (Higher Education Press, In Chinese, 2006)

[4] Jin Xie, and Yong Chen. Mechanical Science and Technology for Aerospace Engineering, vol.29, pp.524-526. (In Chinese, 2000)

[5] Xiangguo Han, Wuyi Chen, and Dingchang Chen. JOURNAL OF BEIJING UNIVERSITY OF AERONVUTICS AND ASTRONAUTICS, vol.28, pp.370-372. (In Chinese, 2002) 